Revista de Investigación de Física 21(1), 182101751 (2018)

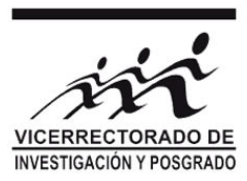

\title{
Impresiones 3D en física aplicada
}

\author{
R. A. Montalvo, A. D. De La Cruz y P. H. Rivera* \\ Facultad de Ciencias Físicas, Universidad Nacional Mayor de San Marcos, Lima, Perú
}

Recibido 21 mayo 2018 - Aceptado 30 junio 2018

\begin{abstract}
En los últimos 10 años, se ha observado una gran revolución tecnológica en la industria aditiva de bajo costo que involucra la impresión 3D para todo tipo de usuarios que abarca desde la propia industria de diseño hasta las aplicaciones diversas que se realizan desde la enseñanza a nivel primario, secundario y universitario hasta el desarrollo de protesis y órganos en la medicina, piezas dentarias en la odontología, artículos para la moda, diseños de arquitectura, piezas y repuestos en la industria automotriz y energía, y obviamente, también en la física aplicada.

En el presente arículo, presentamos nuestra experiencia directa con la impresión 3D, mencionamos detalles de la técnica Fuse Deposit Modelling, FDM, para el cual hemos ensamblado dos impresoras 3D y relatamos nuestro aprendizaje en la impresión de objetos 3D diseñados para un espectrómetro Raman con el programa OpenScad, procesados por el programa Slic3r e impresos usando el $g$-code por las impresoras 3D que hemos ensamblado.
\end{abstract}

Palabras claves: Impresión 3D, filamentos, FDM, STL, g-code..

\section{D printing in Applied Physics}

In the last ten years, it's has been observed a technologic revolution in the low cost additive industry in which is involved the 3D printing open for a broad users universe that range from the same design industry to the diverse applications realized in primary, secondary and university education, and to prosthesis and organs development in medicine, dental pieces in odontology, fashion articles, architecture designs, automotriz industry parts and energy, and obviuosly, also in applied physics.

In the present article, we present our direct experience on 3D printing, discussing about the fuse deposit modelling technique, FDM, for which we assembly two 3D prints and we talk about our 3D printing learning of objects designed for a Raman spectrometer using the OpenScad program, processing with Slic $3 r$ from Prusa and printed with g-code commands in the 3D printers assembled by us.

Keywords: 3D printing, filaments, FDM, STL, g-code..

Desde la fusión nuclear [1] hasta la enseñanza [2], la impresión 3D tiene amplias aplicaciones en la física, sin mencionar las a plicaciones que se están realizando en otras áreas como la medicina, la arquitectura y en la ingeniería en general, en sus diferentes especialidades como la construcción, la industria automotriz y la energía.

Históricamente, en la ingeniería, se han desarrollado tres técnicas de manufactura, estos son, la sustractiva, la formativa y la aditiva. Esta última técnica es la que se ha desarrollado en las cuatro últimas décadas, aunque tradicionalmente en toda la industrial mundial predomina la sustractiva y la formativa por su bajo costo relativo en cuanto a volumen de producción [3].

En este artículo, hacemos una breve descripción de las metodologías desarrolladas en la manufactura aditiva

\footnotetext{
* priverar@unmsm.edu.pe
}

y describimos la experiencia desarrollada en nuestro grupo Lab2D4 sobre la impresión 3D basado en la tecnología Fuse Deposit Modelling, FDM, para la construcción del sistema mecánico de un espectrómetro Raman.

\section{Precedentes}

La impresión 3D es una técnología relacionada a la manufactura aditiva que construye un objeto tridimensional desde un modelo diseñado con la asistencia de la computadora, mediante la adición sucesiva de materiales en las direcciones $x$ e $y$, y capa por capa en la dirección $z$ 4 5].

Desde que Hideo Kodama en 1981 [5] inventó dos métodos de manufacturación aditiva usando polímeros térmi- 
cos fotoendurecidos por radiación ultravioleta, UV, hasta el presente, se han desarrollado varios procesos de manufacturación aditiva que se resumen en los siguientes procesos [3]: (a) Extrusión de materiales, es un proceso consistente en depositar un filamento fundido capa por capa que paulatinamente se va enfriando y endureciendo en el medio ambiente, la tecnología más conocida es el Fuse Deposit Modelling, FDM, también conocido como Fuse Filament Fabrication, FFF; (b) Vat Polimerization, en este proceso se deposita un filamento fundido o una resina líquida que se cura o endurece mediante un haz laser UV, las tecnologías desarrolladas mediante este proceso son el Stereolithography, STL o SLA, y el Direct Light Processing, DLP; ambas tecnologías tienen amplias aplicaciones en Odontología, en la manufactura de protésis dentales [6 7]. y en el área de prótesis y órganos artificiales [8-10]; (c) Powder Bed Fusion, este proceso como lo indica su nombre se tiene una capa de material en polvo sobre el cual incide un haz de laser UV fundiendo el material en zonas específicas que se controlan mediante el computador, las tecnologías desarrolladas en este proceso son el Selective Laser Sintering, SLS; el Direct Metal Laser Sintering, DMLS; el Selective Laser Melting, SLM y el Electron Beam Melting, EBM; (d) MJ, Material Jetting, este proceso de manufactura aditiva consiste en generar gotas de materiales fundidos específicos para el crecimiento del objeto capa a capa, la técnica desarrollada es el Drop On Demand, DOD; (e) BJ, Binder Jetting, es el proceso que consiste en inyectar un fluido ligante que permite enlazar regiones de una capa en polvo bajo la acción de un laser UV; (f) Direct Energy Deposition, en este proceso se incide un haz laser del infrarrojo, IR, sobre un material que debe ser fundido al mismo tiempo en que es depositado, las técnicas desarrolladas son el Laser Engineering Net Shaping, LENS y el Laser Based Metal Deposition LBMD; (g) Sheet Lamination, en este proceso las capas separadas de materiales son aglomeradas capa a capa para obtener el objeto diseñado, las técnicas más usuales son el Ultrasonic Additive Manufacturing, UAM, y el Laminated Object Manufacturing, LOM, [3].

En el 2009, expiró la patente de la tecnología asociada con el FDM, este hecho permitió a la comunidad tecnológica internacional, luego de la gran crisis financiera del 2008, crear proyectos abiertos en base a esta tecnología. Surgieron empresas inicialmente asociadas a esta tecnología como Makerbot [11], Ultimaker [12], 3D Systems 13. (1986), Prusa Research [14] y otros [15]. En el interín surgió un proyecto en la que una maquina 3D se autoreplique [17] a muy bajo costo. Las impresoras 3D de marca registrada superan los dos mil dólares para los tamaños pequeños $15 \mathrm{~cm} \times 15 \mathrm{~cm}$. La participación intensiva de China y Europa del Este ha sido importante en la disminución de los precios de las impresoras 3D hasta menos de 500 dólares en el mercado local. En el último año, se pueden encontrar, también en el mercado local, impreso- ras 3D fabricadas por la Ultimaker, Formlabs, Sintratec y Bigrep 18.

\section{Impresora 3D}

En nuestro grupo Lab2D4 hemos implementado dos impresoras 3D Tevo [19] basados en la tecnología de Fuse Deposit Modelling que consiste en la extrusión térmica de un filamento polímerico que al ser fundido por un calefactor pasa a través de un nozzle con un diámetro de $0.4 \mathrm{~mm}$ y se deposita línea a línea con diámetros que abarcan desde $0.1 \mathrm{~mm}$ hasta $1.0 \mathrm{~mm}$ en las direcciones $x$ e $y$, y capa por capa en la dirección $z$ donde dichos movimientos son realizados por cuatro motores de paso, tres para los ejes $x, y$ y $z$, y el cuarto motor que mueve el filamento polimérico hacia el sistema calefactor que funde el polímero y por el nozzle sale el filamento fundido que se deposita en el material que se ha depositado en el paso previo y que se ha enfriado a temperatura ambiente. Los motores de paso son controlados por una placa Arduino Mega [16] con el chip Atmega 2560 y el Ramps 1.4 de la RepRap |17] que incorporan en su arquitectura los sensores de paso de los motores, los sensores y el controlador de la corriente del calefactor y de la cama caliente, la plataforma horizontal donde se construye el objeto 3D que es estrictamente el plano $X Y$ que se mueve. La placa electrónica se denomina MKS Base V1.5 de la Rerap, ver Figura1.

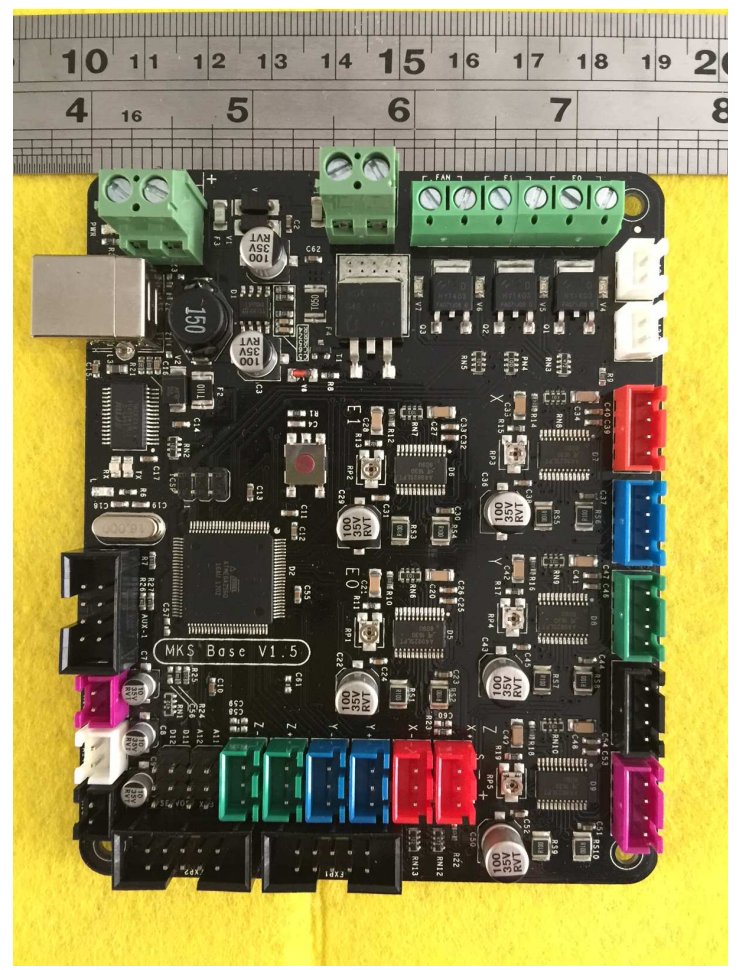

Figura 1: Se muestra la placa controladora MKS Base V1.5 que contiene el arduino 2560 y el Ramps 1.4 de la Reprap [17]. 
La parte mecánica de las impresoras 3D están construídas en aluminio o acero inoxidable, la elección de este detalle incrementa el costo. Para la elaboración de objetos 3D para su uso en biología, odontología o medicina es preferible el acero inoxidable para el mantenimiento de su limpieza clínica. La mayoría de los diseños mecánicos consisten de una plataforma horizontal denominada como cama caliente, donde se forman los objetos $3 D$, que se mueve a través de rodamientos en la dirección y y el movimiento en $x$ es realizado por el extrusor de los filamentos que también usa rodamientos para disminuir la vibración y el movimiento en $z$ es realizado por la barra horizontal que sostiene al extrusor. En otros diseños la plataforma horizontal realiza el movimiento en $z$ y el extrusor realiza el movimiento en $x$ y la barra horizontal que sostiene el extrusor realiza el movimiento en $y$ o viceversa. Para el primer diseño, es interesante observar que el movimiento vibracional producido por el desplazamiento de la plataforma horizontal en la dirección y genera una oscilación mecánica pequeña, la cual es bastante menor cuando la plataforma se desplaza en la dirección $z$. Desafortunadamente, este último diseño ha sido patentado por la UItimaker y los sistema abiertos 3D usan el primer diseño. Esas oscilaciones son compensadas via el código g [20] administrando el movimiento del filamento en el extrusor. La parte mecánica de la impresora $3 \mathrm{D}$ se observa en la Figura 2 La versatilidad de las impresoras 3D dependen de su fortaleza mecánica.

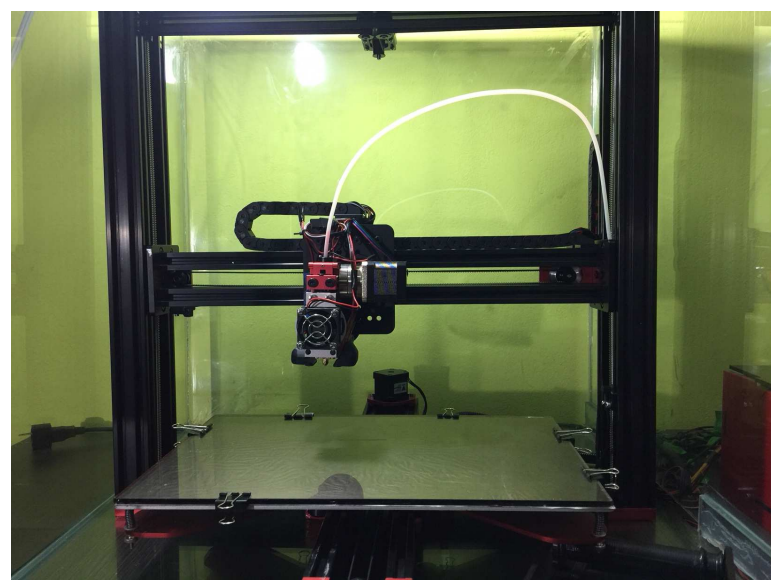

Figura 2: Se muestra la parte mecánica de la impresora 3D del grupo Lab2D4.

En la Figua 2 se muestra el marco principal de aluminio que representa el eje $x$ del movimiento del extrusor. En la parte central de la Figura 2 se muestra el eje horizontal que se mueve en el eje $z$ y sobre el cual se instala el extrusor de color rojo que se mueve paralelo a la barra a lo largo del eje $x$. El extrusor comprende, en el lado derecho, el motor de paso que mueve el filamento, en la parte inferior, el ventilador que enfría el calefactor que se encuentra detrás del ventilador y por donde pasa el filamento a fundir y el nozzle de bronce en la parte inferior por donde sale el material fundido. En lado derecho, desde el extrusor se observa el tubo blanco de neopreme a través del cual se conduce el filamento desde la bobina de filamento que no se muestra en la figura. En la parte inferior de la figura, se observa la cama caliente cubierta de vidrio y sujetada con cuatro clips. Su movimento es en el eje $y$, hacia el observador. En la parte central al fondo se muestra la silueta del motor de paso que mueve la cama caliente a lo largo del eje $y$. En el extremo derecho de la barra horizontal donde se mueve el extrusor se encuentra escondido el motor de paso que mueve el extrusor en la dirección $x$. Y en la parte superior y central de la barra horizontal se muestra la silueta del motor de paso responsable del movimiento en el eje $z$.

\section{El extrusor}

La parte más importante de una impresora 3D es el diseño del extrusor de filamentos porque de acuerdo a su versatilidad puede imprimir más de un filamento y mas de un tipo de filamento. En la barra horizontal que se mueve en el eje $z$ se encuentra el espacio necesario para colocar un extrusor de acuerdo a las necesidades propias del proyecto a imprimir. Cabe resaltar que, cuando más grande es el extrusor menor es el volumen de impresión.

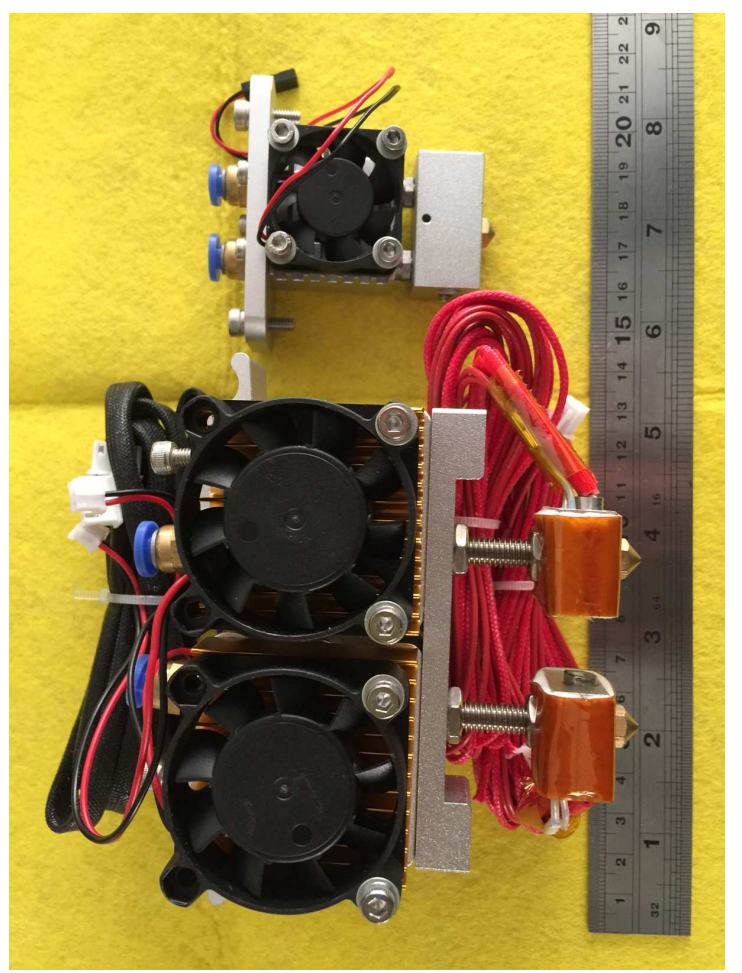

Figura 3: Se muestra dos diseños de extrusores de filamento para una impresora 3D. 
En la figura 3 se muestra dos diseños de extrusores que permiten la impresión simultánea de dos filamentos de diferentes tipos o colores si son del mismo tipo o un filamento para el objeto 3D y el otro filamento soluble en agua que se usa como soporte para el objeto 3D. El extrusor más pequeño, en la parte superior de la figura 3 permite el ingreso de dos filamentos a través de dos conectores neumáticos al calefactor y solo tiene un nozzle para la salida secuencial o simultánea de los dos filamentos, para este extrusor los motores que empujan los filamentos se instalan en el marco del soporte mecánico. En la parte inferior de la figura 3 se muestra un diseño de dos extrusores en una sola pieza, con dos motores de paso independientes que empujan el filamento y salen a través de los nozzles independientes. Aquí la impresión se tiene que sincronizar y calibrar via el uso del código G [20]. Las impresoras 3D de la Ultimaker, el modelo S5, poseen extrusores que permiten el ingreso de hasta 3 filamentos.

Los extrusores están diseñados para imprimir los siguientes tipos de filamentos: (1) ABS, Acrylonitrile Butadiene Styrene, es el primer filamento que apareció en el mercado, se caracteriza por tener una buena estabilidad mecánica por su rigidez y resistencia mecánica, pero es fácilmente soluble en acetona, por esta razón, se usa vapores de acetona para dar un acabado brillante al objeto 3D. Se usa bastante en diseños de estructuras mecánicas resistentes que no estén en contacto con acetona, la temperatura de fusión está entre $190-230^{\circ} \mathrm{C}$, dependiendo del color y del fabricante. Es necesario el uso de la cama caliente entre $70-100^{\circ} \mathrm{C}$ y una temperatura ambiente de $60^{\circ} \mathrm{C}$, por ello es necesario un ambiente cerrado para la impresora 3D, los vapores que se expele durante el proceso de impresión son tóxicos, por tanto, se debe evitar todo contacto durante el proceso de impresión. Se sugiere el uso de una cámara web para observar el desarrollo del proceso de impresión. Se recomienda el uso de cintas de pintor azul sobre el vidrio de la mesa caliente y la aplicación de laca para el cabello sobre la superficie donde se va imprimir el objeto $3 \mathrm{D}$ y se adhiera a la superficie, sino el objeto se deforma conforme aumenta la altura, este es el principal defecto de este filamento, no es recomendable para objetos de gran volumen. (2) PLA, PolyLactic Acid, es el filamento, actualmente, el más comercial debido a su versatilidad respecto al ABS. Su temperatura de impresión está entre $180-210^{\circ} \mathrm{C}$, dependiendo del color y del fabricante. Para objetos grandes no tiene competencia si la impresión se realiza en un ambiente con temperatura constante de $40-60^{\circ} \mathrm{C}$, y no es necesario el uso de cama caliente, ni el uso de pegamentos para que el objeto se adhiera. Este material se adhiere bien sobre el vidrio colocado sobre la cama. (3) PLA duro, Tough PolylActic Acid, es el filamento PLA con las mismas propiedades mecánicas del ABS, pero no posee los defectos de deformación de los objetos 3D impresos con ABS. (4) PET, PolyEthylene Terephthalate, es un filamento con las mismas propiedades de impresión del PLA y con las propiedades mecánicas del ABS, como el PET es un material bastante usado en las industrias de las bebidas y de los textiles, es reciclable y amistoso para el medio ambiente. Cuando se imprime no despide olores ni son tóxicos. Para la impresión se usa la cama caliente a $80^{\circ} \mathrm{C}$ y la temperatura del extrusor debe estar entre $220-260^{\circ} \mathrm{C}$, dependiendo del fabricante. No es necesario usar cinta del pintor azul, solo hechar un poco de laca para el cabello sobre la superficie de vidrio de la cama caliente para objetos de gran volumen. Debido al gran enlace molecular que posee el PET se adhieren bien las capas verticales sin deformarse 21]. (5) Nylon, Polyamide, es un filamento que, recientemente, ha salido al mercado, y se utiliza para imprimir objetos 3D que van a estar expuestos a ambientes corrosivos, son durables, flexibles, baja fricción, tienen una alta razón de rigidez sobre peso y está diseñado para construir herramientas para la propia impresión 3D. Nuestro grupo Lab2D4 aún no ha impreso este material. (6) CPE, ColoPolyEster, es un filamento químicamente resistente, duro y muy estable dimensionalmente. Muy usado en prototipos y partes mecánicas de dispositivos. Alta resistencia térmicas y a altos impactos. (7) PVA, PolyVinil Alcohol, es el filamento soluble en agua usado como soportes de estructuras aéreas de los objetos 3D que se imprimem con filamentos ABS o PLA u otros. (8) HIPS, High Impact PolySterene, este filamento es para objetos que soporten una resistencia de alto impacto incluso a bajas temperaturas, esencialmente tienen estructuras repetitivas de estireno y butadieno. Nuestro grupo Lab2D4 ha adquirido unas bobinas de este material que incluyen fibra de carbono y grafeno [22], la temperatura de impresión es de $230^{\circ} \mathrm{C}$, no necesita cama caliente. (9) TPE, ThermoPlastic Elastomer o TPU, Thermoplastic PolyUrethane, estos filamentos son de gran interés por su flexibilidad, sirve para imprimir sellos o contactos flexibles como interface entre dos superficies metálicas. Cualquiera sea la superficie de contacto entre superficies se diseña y se imprime. La temperatura de impresión es de $210-240^{\circ} \mathrm{C}$ con la cama caliente a la temperatura de $20-70^{\circ} \mathrm{C}$, que depende esencialmente del fabricante y del color. Para filamentos oscuros se recomienda añadir $5^{\circ} \mathrm{C}$ respecto de los filamentos claros. El extrusor recomendable para imprimir los dos últimos filamentos HIPS y TPE o TPU es el mostrado en la parte inferior de la figura 3 donde los motores de paso están muy próximos $(\sim 2 \mathrm{~cm})$ al calefactor del extrusor.

\section{Diseño de objetos}

Para imprimir un objeto en una impresora 3D, antes debemos diseñarlo de acuerdo a las ideas desarrolladas para construir un dispositivo. Para ello necesitamos de los programas CAD, Computer Aided Design, que traducido al español es el diseño asistido por computadora. En el 
Linux, existen varios programas para el diseño de objetos, como QCad [23], FreeCAD [24], Open CASCADE Technology [25], CyCAS 26] y CityEngine [27. para arquitectos, y el BRL-CAD [28], el cual es uno de los mejores programas de diseño que se ha desarrollado en los últimos 20 años por la comunidad de internet pero Michael Jhon Muuss, fue el creador original de este proyecto mientras trabajaba en el U.S. Army Research Laboratory's. Él también contribuyó en el desarrollo del protocolo TCP-IP que dió origen a la Internet, murió relativamente joven en noviembre del 2000 [28].

Pero, el programa especializado para diseñar dispositivos mecánicos para impresión $3 \mathrm{D}$, versátil y fácil de usar es el OpenScad [29] que abre archivos DXF producido por los programas CAD y graba en formato STL, Standard Triangle Language, el cual es un formato de archivos que describe solamente la geometría de los objetos 3D, fue desarrollada por la empresa 3D Systems, la primera empresa en tecnología 3D fundada por Chuck Hull quien tuvo la patente de la tecnología SLA, StereoLitography en 1986 [30], que consiste en la impresión 3D de resinas líquidas que son curadas por luz UV o un laser UV, discutida en una sección anterior. En la figura 4 se muestra el frontend del OpenScad mostrando el diseño del espectrómetro Raman que estamos construyendo.

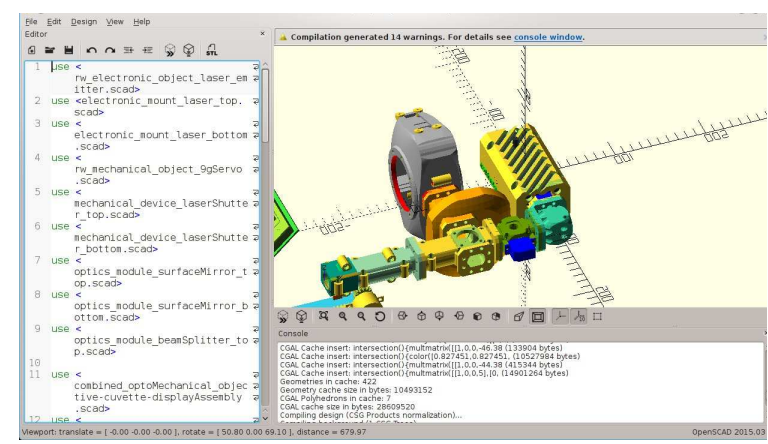

Figura 4: Se muestra el frontend del OpenScad con el diseño del espectrómetro Raman que estamos construyendo.

Los archivos stl generados por el OpenScad deben ser traducidos al código de programación de control numérico g-code, el cual es el lenguaje de programación estándard del Control Numérico por Computadora, CNC, con el cual las máquinas industriales son controladas para realizar el proceso de fabricación de piezas, este lenguaje fue diseñado por el MIT, Massachusetts Institute of Technology, para su uso en todas las máquinas o herramientas automáticas. Como la impresora 3D es una máquina automática controlada por el g-code, las instrucciones para la impresión están desarrolladas numéricamente mediante el Slic3r [31. que es un programa libre realizado en Python y producido por la Prusa Research [14], empresa creada por Josef Prusa, quién diseñó y construyó la primera impresora abierta 3D para filamentos, el Original PRUSA i3 MKO y
MK1. Los archivos st/ para las partes de estas impresoras lo pueden encontrar en el sitio de la Prusa Research [14]. En la figura 5 , se muestra un segmento del espectrómetro Raman procesado en el Slic3r para generar el archivo g-code que usará la impresora 3D para construir el objeto 3D.

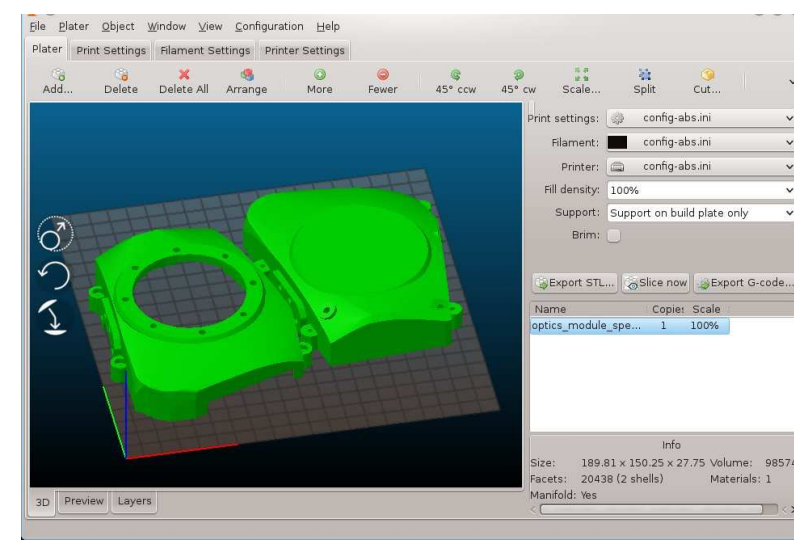

Figura 5: Se muestra el frontend del Slic3r de la Prusa Research con el diseño de un segmento del espectrómetro Raman que estamos construyendo. Este prgrama transforma el formato stl al g-code.

\section{Resultados y discusión}

Luego del largo proceso de ensamblaje, alineación mecánica del sistema, preparación del sistema de aislamiento térmico de la impresora 3D, reconfiguración del sistema de control electrónico fuera del aislamiento térmico, alineamiento y calibración de la cama caliente respecto al movimiento del extrusor, las correcciones del firmware del Arduino para calibrar el movimiento del filamento en el extrusor y la determinación de las temperaturas idóneas de calefactor del extrusor y de la cama caliente, obtuvimos nuestras primeras impresiones de óptima calidad, después de resolver diferentes problemas de configuración y reconfiguración. En ese interín, hemos observado que determinados diseños requieren de soportes y el gran problema es determinar el programa que construye los mejores soportes. El Slic3r de Prusa Research [31], crea soportes bastante densos para los objetos, usando el filamento PVA para los soportes y luego de terminado la impresión, el objeto 3D queda impecable sin los soportes al ser sumergido en agua pues los filamentos PVA son solubles en el agua, como ya se mencionó previamente. Pero los filamentos PVA en el mercado local son inexistentes, entonces la alternativa es usar programas como el Cura de la Ultimaker [32. y el Meshmixer de la Autodesk Inc. [33. ambos son gratuitos. El Meshmixer construye los soportes menos densos y más adecuados de acuerdo al diseño del objeto. Este procedimiento permite el ahorro de filamento cuando 
se tiene un solo extrusor en la impresora $3 \mathrm{D}$, puesto que se usa el mismo filamento tanto para el objeto como para los soportes. En la figura 6 se muestra algunos componentes del espectrómetro Raman elaborados por las impresoras 3D ensambladas en nuestro laboratorio del grupo Lab2D4.
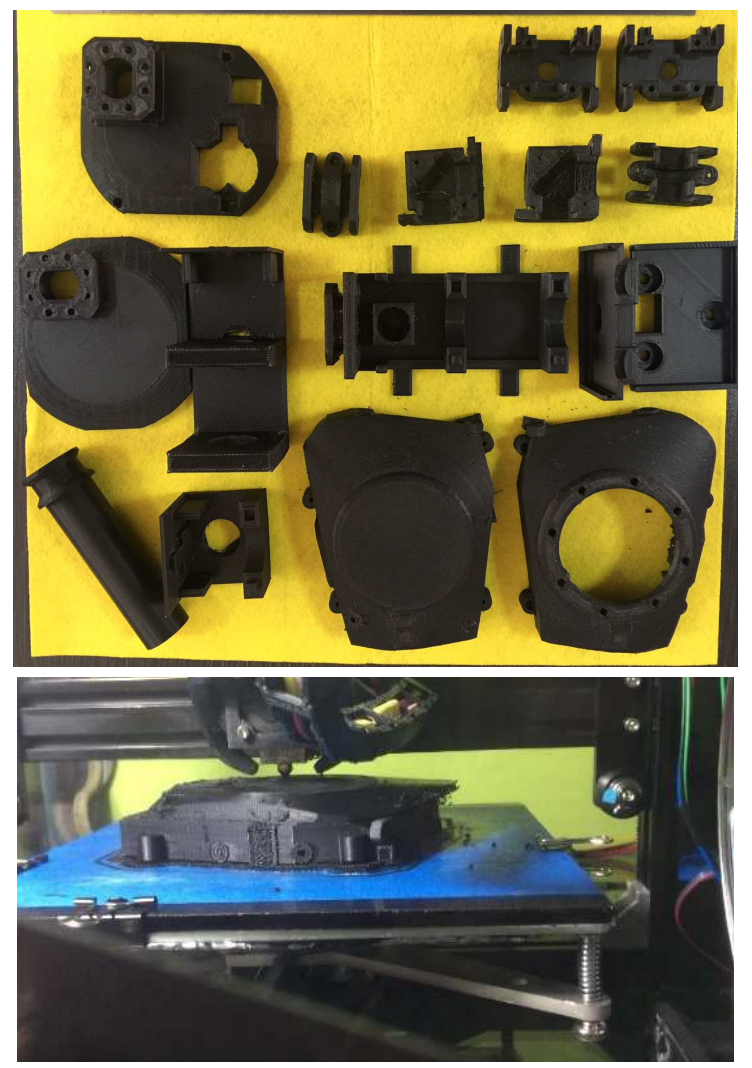

Figura 6: Se muestran algunos componentes del espectrómetro Raman elaborados por las impresoras 3D del grupo Lab2D4, en la parte superior. $Y$ en la parte inferior, presentamos una instantánea de la impresión.

El área de la impresión 3D tiene amplias aplicaciones en física, el principal es la investigación en la mezcla de polímeros con materiales diversos en polvo tales como la madera, la arcilla, metales magnéticos, metales conductores, semiconductores, superconductores, ópticos y ferroe- léctricos para construir piezas que posean determinadas propiedades físicas del material en polvo después de su procesamiento. Sobre esto, existen varios ejemplos en la literatura con resultados que han sido obtenidos en los diferentes laboratorios del mundo. Uno de los más recientes es el uso de la tecnología 3D, el Selective Laser Melting en la elaboración de los aceros ferríticos/martensíticos de reducida activación neutrónica para construir los breeding blanket para el International Thermonuclear Experimental Reactor que se está construyendo en Francia [1], este trabajo fue realizado por un grupo del Institute of Nuclear Energy Safety Technology de China, con un material que fue desarrollado hace más de diez años en China, denominado China Low Activation Martensitic. Sobre este material en polvo y depositado uniformemente sobre la cama incide un haz laser de $400 \mathrm{~W}$ que se propaga a través de una fibra óptica para formar las microstructuras de $60 \mu \mathrm{m}$. La cama del sistema Renishaw SLM Realizer AMS250 tiene un volumen efectivo de trabajo de $250 \mathrm{~mm} \times 250 \mathrm{~mm}$ $\times 300 \mathrm{~mm}$. En nuestro caso, nuestras impresoras 3D tienen un volumen de trabajo efectivo de $200 \mathrm{~mm} \times 200 \mathrm{~mm}$ $\times 220 \mathrm{~mm}$ y de $220 \mathrm{~mm} \times 330 \mathrm{~mm} \times 320 \mathrm{~mm}$. Finalmente, para los interesados en la tecnología SLM o SLA, existe un proyecto sobre un sistema abierto [30] que puede ser construído a muy bajo costo, pero las resinas que se usan para la fabricación de los objetos $3 \mathrm{D}$ son relativamente caros.

\section{Conclusiones}

Hemos tenido una buena etapa de aprendizaje sobre la tecnología Fuse Deposit Modelling durante el proceso de ensamblaje, calibración y programación de una impresora 3D que imprime filamentos ABS, PLA, PVA, HIPS y flexibles. Esta es una valiosa experiencia dentro de la metodología DIY, Do It Yourself, que ha surgido en las universidades del mundo como un efecto de la gran crisis financiera del 2008. Como objetivo principal, hemos construído la parte mecánica de un espectrómetro Raman y el sistema de calibración del espectrómetro. El know-how del diseño con el OpenScad y procesamiento antes de la impresión propiamente dicha con el Slic3r o el Cura o el Meshmixer son tareas que recomendamos a los lectores antes de imprimir un objeto 3D.

\section{Referencias}

[1] Bo Huang, Yutao Zhai, Shaojun Liu y Xiaodong Mao; J. Nuclear Materials 500, 33 (2018). https: //doi.org/10.1016/j.jnucmat. 2017. 12.011
[2] https://www .thingiverse.com/explore/new est/learning/physics-and-astronomy

[3] Ben Redwood, Filemon Schöffer y Brian Garret; The 3D Printing Handbook: Technologies, design and applications, 3D Hubs B. V., Amsterdam (2017).

[4] Mohammad Taufik y Prashant K. Jain; Int. J. Ma- 
nufacturing Technology and Management 27, 43-73 (2014).

[5] https://en.wikipedia.org/wiki/3D_printing

[6] http://www .medicalexpo.es/fabricante-med ical/impresora-3d-odontologia-26323.html

[7] http://zenith3d.co.kr/eng/

[8] N. H. Cohrs, A. Petrou, M. Loepfe, M. Yliruka, C. M. Schumacher, A. Xavier Kohll, C. T. Starck, M. Schmid Daners, M. Meboldt, V. Falk y W. J. Stark; Artificial Organs 41, 948 (2017).

[9] https://www.openbionics.com

[10] https://3dprint.nih.gov/collections/pros thetics

[11] https://www.makerbot.com

[12] https://ultimaker.com

[13] https://www.3dsystems.com

[14] https://www.prusa3d.com

[15] https://en.wikipedia.org/wiki/List_of_ 3D_printer_manufacturers

[16] https://www.arduino.cc

[17] http://reprap.org

[18] https://ww. cadillo3dstore.pe
[19] https://tevo3dprinterstore.com

[20] https://reprap.org/wiki/G-code

[21] https://e3d-online.com/filaments-3d-print ing/spoolwoks/edge

[22] http://blackmagic3d.com

[23] https://www.qcad.org

[24] https://www.freecadweb.org

[25] https://www. opencascade.com

[26] http://www.cycas.de

[27] https://www.esri.com/

[28] http://brlcad.org

[29] http://www.openscad.org

[30] https://en.wikipedia.org/wiki/Stereo lithography

[31] https://ww. prusa3d.com/slic3r-prusa-edi tion

[32] https://ultimaker.com/en/products/ultima ker-cura-software

[33] http://www.meshmixer.com

[34] https://www.instructables.com/id/Build-aLaser-3D-Printer-Stereolithography-at-Ho/ 
